\title{
Edge Computing-based Differential Positioning Method for BeiDou Navigation Satellite System
}

\author{
Lina Wang ${ }^{1,2,3^{*}}$, Linlin $\mathrm{Li}^{1}$, Rui Qiu ${ }^{1}$ \\ ${ }^{1}$ School of Computer and Communication Engineering, University of Science and Technology Beijing, 100083, \\ Beijing, P. R. China \\ ${ }^{2}$ Beijing Engineering and Technology Research Center for Convergence Networks and Ubiquitous Services, \\ University of Science and Technology Beijing, Beijing 100083, China \\ ${ }^{3}$ Beijing Key Laboratory of Knowledge Engineering for Materials Science, Beijing, China \\ [e-mail: wln_ustb@126.com, follow1993@outlook.com, Naliduo1221@outlook.com] \\ *Corresponding author: Lina Wang
}

Received December 27, 2017; revised July 14, 2018; accepted September 8, 2018; published January 31, 2019

\begin{abstract}
BeiDou navigation satellite system (BDS) is one of the four main types of global navigation satellite systems. The current system has been widely used by the military and by the aerospace, transportation, and marine fields, among others. However, challenges still remain in the BeiDou system, which requires rapid responses for delay-sensitive devices. A differential positioning algorithm called the data center-based differential positioning (DCDP) method is widely used to avoid the influence of errors. In this method, the positioning information of multiple base stations is uploaded to the data center, and the positioning errors are calculated uniformly by the data center based on the minimum variance or a weighted average algorithm. However, the DCDP method has high delay and overload risk. To solve these problems, this paper introduces edge computing to relieve pressure on the data center. Instead of transmitting the positioning information to the data center, a novel method called edge computing-based differential positioning (ECDP) chooses the nearest reference station to perform edge computing and transmits the difference value to the mobile receiver directly. Simulation results and experiments demonstrate that the performance of the ECDP outperforms that of the DCDP method. The delay of the ECDP method is about 500ms less than that of the DCDP method. Moreover, in the range of allowable burst error, the median of the positioning accuracy of the ECDP method is $0.7923 \mathrm{~m}$ while that of the DCDP method is $0.8028 \mathrm{~m}$.
\end{abstract}

Keywords: BeiDou satellite, Navigation system , Differential positioning, Edge computing

This paper is supported by the National Natural Science Foundation of China under Grants No. 61701020 and No. 61170014. 


\section{Introduction}

The BeiDou navigation satellite system (BDS) developed by China can provide users with high precision as well as all-weather and real-time positioning and navigation [1-3]. The BDS has a wide range of applications in military and civilian areas. It requires a rapid response and high precision. Consequently, it is necessary to investigate the positioning algorithm for the BDS, thereby impoving the compititiveness and promoting the development of the BDS.

Due to satellite ephemeris errors, clock errors, tropospheric errors and many other factors, deviations often exist in satellite positioning, making it difficult to meet the needs of high-precision positioning [4]. Differential positioning technology has been introduced into the BeiDou system to eliminate the influence of position deviation. Due to its ability to offset the public error of mobile and reference stations, the differential positioning algorithm has become the preferred correction technique and is now globally used in various applications [5].

In the original data center-based differential positioning (DCDP) method, each base station transmits the observed data to the data center continuously, the data center can obtain its synthesized difference value in view of the minimum variance or the weighted average algorithm according to multiple reference station observations [6]. After the error deviation is calculated, the data center broadcasts the deviation data to mobile receivers. When the equipment receives the satellite signal, the mobile receivers add the difference value to obtain final three-dimensional coordinate, improving positioning accuracy. The accurate position of the receiver can be determined according to the difference value and the distance between the satellite and the user receiver [7]. However, the technology uses the data center computation to transmit the deviation data. Although this method provides many benefits to users, such as increased data handling and better computing ability, several challenges remain when using this method [8].

One of the challenges is that the network bandwidth that carries data to and from a data center does not increase as user positioning demand increases [9]. As a result, with greater amounts of data being generated by users, the core network wastes large amounts of bandwidth. Another challenge arises when users require shorter response time and more efficient processing from the system for delay-sensitive applications. Furthermore, use of the data center to process the positioning information will also raise privacy concerns [10]. The most important challenge is that the "anti-fragility" ability of the DCDP method is very low. Once the data center is under attack, the performance of the system would be affected.

To solve the above problems, the paper introduces edge computing into the BDS and proposes a novel method called edge computing-based differential positioning (ECDP). The ECDP method offloads various computing tasks to the network edge, which allows the use of its services with low delay and high reliability. Edge computing can offload the pressure on the core network resulting from processing, storage, caching, and load balancing [11].

The scheme is designed for the case in which at least two reference stations are within the positioning range. Specifically, under the scheme, the progress of sending the correction to the data center is removed, which is time consuming and results in data transmission delay. Thus, this paper proposes using the reference station closest to the mobile station to compute and transmit data directly to the mobile station. In this manner, the ECDP will enable a low delay positioning process and share the pressure on the data center. And what's more, "anti-fragility" ability of the BDS will be greatly enhanced. 
The remainder of this paper is organized as follows. Section 2 presents the original DCDP algorithm model and formulates the calculation progress of DCDP. Section 3 describes the ECDP method. Section 4 presents the experimental results and analyses. Finally, Section 5 concludes the paper.

\section{DCDP Positioning Model And Formulation}

\subsection{Principle of the Pseudo-range Difference}

Differential positioning technology can be divided into four types of algorithms according to the information used: position difference, pseudo-range difference, phase smooth pseudo-range difference or carrier phase difference [12-14]. The four differential positioning techniques work in a similar manner. The mobile station corrects the positioning result by receiving the difference value from the reference station, which can improve the positioning accuracy. However, these four algorithms have their own characteristics and their respective application scenarios. The comparisons of four differential algorithms are listed in Table 1.

Table 1. Comparison of four differential algorithms

\begin{tabular}{|c|c|c|c|}
\hline Classification & Advantage & Disadvantage & Scene \\
\hline $\begin{array}{c}\text { Position Difference } \\
\text { algorithm }\end{array}$ & $\begin{array}{c}\text { simple calculation; } \\
\text { good compatibility }\end{array}$ & $\begin{array}{c}\text { low positioning } \\
\text { accuracy }\end{array}$ & $\begin{array}{c}\text { Public Location } \\
\text { Service }\end{array}$ \\
\hline $\begin{array}{c}\text { Pseudo-range } \\
\text { Difference algorithm } \\
\text { satellites is effective; } \\
\text { no need to convert } \\
\text { coordinate system }\end{array}$ & $\begin{array}{c}\text { accuracy decreases } \\
\text { with the distance from } \\
\text { base station to mobile } \\
\text { station }\end{array}$ & $\begin{array}{c}\text { Location Based Service } \\
\text { (LBS) }\end{array}$ \\
\hline $\begin{array}{c}\text { Phase Smooth } \\
\text { Pseudo-range }\end{array}$ & $\begin{array}{c}\text { long static observation } \\
\text { time; phase } \\
\text { positioning accuracy } \\
\text { observations cannot } \\
\text { have large cycle slip }\end{array}$ & $\begin{array}{c}\text { Weather Forecast; } \\
\text { Marine Mapping }\end{array}$ \\
\hline $\begin{array}{c}\text { Carrier Phase } \\
\text { Difference algorithm }\end{array}$ & high accuracy & $\begin{array}{c}\text { long static observation } \\
\text { time; cannot appear } \\
\text { code error or satellite } \\
\text { lockout }\end{array}$ & $\begin{array}{c}\text { Engineering } \\
\text { Measuring; } \\
\text { Ground Subsidence } \\
\text { Monitoring }\end{array}$ \\
\hline
\end{tabular}

Although the DCDP and ECDP methods are applicable to all above differential techniques, due to space limitations and demand of civil development, this paper considers the most widely used and most mature type, namely, the pseudo-range difference.

The pseudo-range difference is one of the most mature BeiDou differential positioning technologies. The pseudo-range is the distance between the BeiDou receivers and all visible satellites. In addition, the distance between the true coordinates of each receiver and the satellite is called the true distance. Then, the pseudo-range difference between the measurement distance and true distance can be obtained. Within the positioning area, multiple reference stations that constitute the continuously operating reference stations (CORS) system send the pseudo-range difference values to the data center. The data center computes the final difference of the pseudo-range synthetically, and then broadcasts the final data to the mobile receivers. The mobile station corrects the pseudo-range by adding the pseudo-range difference value. Finally, the user receiver calculates the more accurate coordinate values based on the corrected pseudo-range. The model of the DCDP system is shown in Fig. 1. 


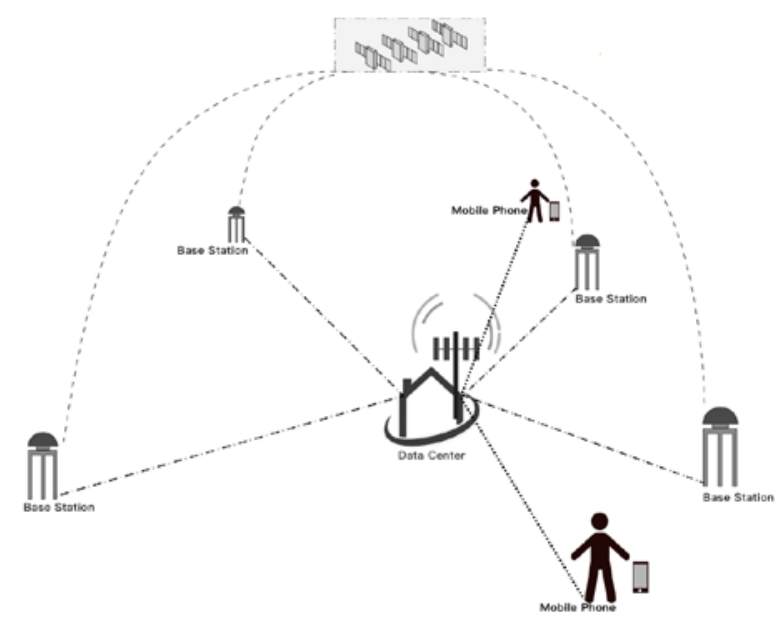

Fig. 1. DCDP system model

\subsection{DCDP Method}

The pseudo-range can be obtained by specifying the time difference between the satellite and user receiver, multiplied by the speed of light. Furthermore, the pseudo-range must consider various errors, such as the satellite ephemeris error, clock error, and tropospheric error. The equation of pseudo-range can be expressed as (1) [12].

$$
\rho_{u}^{k i}=\gamma+\delta t_{u}-\delta t^{i}+I+T+\varepsilon_{\rho}
$$

where $\gamma$ represents the geometric distance of the receiver, $\delta t_{u}$ and $\delta t^{i}$ represent the clock difference and satellite clock errors caused by the amount of pseudo-range changes, respectively, $I$ and $T$ denote the pseudo-range change caused by ionosphere and troposphere, respectively, and $\varepsilon_{\rho}$ is the sum of errors not directly reflected in the equation.

The specific coordinates of satellites can be calculated based on the BeiDou satellite ephemeris files. Thus, let $\left(x_{s}{ }^{i}, y_{s}{ }^{i}, z_{s}{ }^{i}\right)$ denote the coordinates of the visual satellites and $\left(x_{r}^{j}, y_{r}^{j}, z_{r}^{j}\right)$ denote the precise coordinates of the reference station. Then, the true distance from the visual satellites to the reference station is

$$
R^{j i}=\sqrt{\left(x_{r}^{j}-x_{s}^{i}\right)^{2}+\left(\mathrm{y}_{r}^{j}-y_{s}^{i}\right)^{2}+\left(\mathrm{z}_{r}^{j}-z_{s}^{i}\right)^{2}}
$$

According to the least squares algorithm, the reference stations are obtained as $\left(\hat{x}_{r}^{j}, \hat{y}_{r}^{j}, \hat{z}_{r}^{j}\right)$. Thus, the pseudo-range $\rho^{i}$ from the reference station to the satellites is

$$
\rho^{j i}=\sqrt{\left(\hat{x}_{r}^{j}-x_{s}^{i}\right)^{2}+\left(\hat{y}_{r}^{j}-y_{s}^{i}\right)^{2}+\left(\hat{z}_{r}^{j}-z_{s}^{i}\right)^{2}}
$$

The pseudo-range includes various errors. To reduce the impact of these errors, according to the measured receiver coordinates, the deviation of the pseudo-range $\Delta \rho^{j i}$ and its rate of change $\Delta \rho^{j i}$ can be obtained as follows [15]:

$$
\Delta \rho^{j i}=R^{j i}-\rho^{j i}, \Delta \dot{\rho}^{j i}=\frac{\Delta \rho^{j i}}{\Delta t}
$$


In the DCDP method, multiple reference stations send $\Delta \rho^{j i}$ and $\dot{\bullet}^{j i}$ to the data center continuously. There are several algorithms that the data center can be used to calculate the integrated result. One algorithm is the weighted average algorithm. In the positioning area, there are multiple reference stations with different distances and different ages of use. Thus, these stations should have unequal weights in the calculation. A more reliable reference station is given a greater weight, whereas an unreliable reference station is given a low weight or, in some cases, no weight. In addition, the principle of minimum variance should be applied. This algorithm uses the output variance as the adjustment index, and the result is transmitted to the user receiver when the variance is minimum. The adjustment calculation is a method of probability theory that uses $n$ equations to solve $m$ unknowns $(n>m)$ [16]. The weighted average algorithm is adopted in this paper. Then, the data center transmits the final $\Delta \rho^{j i}$ and $\dot{\Delta}_{\dot{\rho}}^{j i}$ results to the users.

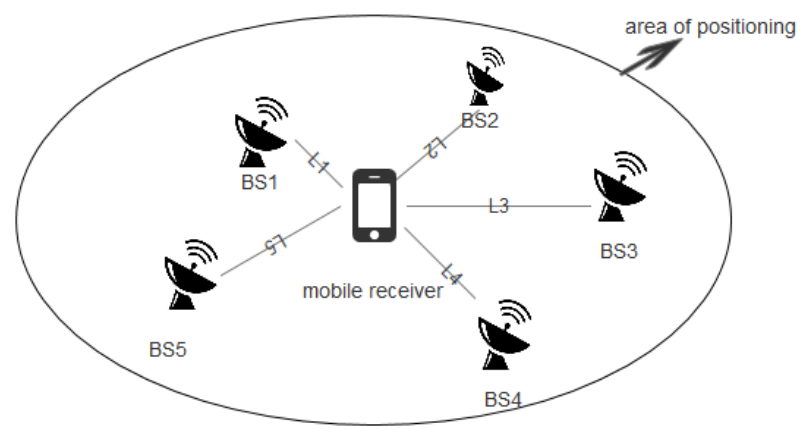

Fig. 2. Distribution of multiple base stations

To obtain the differential data of several reference stations, data are collected according to the distribution shown in Fig. 2. Based on the weighted average principle, the weight number is inversely proportional to the distance, and the final result is shown in (5).

$$
\begin{aligned}
\Delta \rho_{c}^{j i} & =\frac{1}{\sum_{1}^{N} \frac{1}{L_{m}}}\left(\sum_{1}^{N} \frac{1}{L_{m}} \Delta \rho_{c m}^{j i}\right), \\
\Delta \dot{\rho}_{c}^{j i} & =\frac{1}{\sum_{1}^{N} \frac{1}{L_{m}}}\left(\sum_{1}^{N} \frac{1}{L_{m}} \Delta \dot{\rho_{c m}}\right)
\end{aligned}
$$

where $N$ is the number of reference stations in the positioning area and $L_{m}$ is the distance from the mobile receiver to base station $m$.

The mobile station corrects its measured pseudo-range with the pseudo-range correction value.

$$
\rho=\rho_{u}^{k i}+\Delta \rho_{c}^{j i}+\Delta \rho_{c}^{\dot{j i}}\left(t-t_{0}\right)
$$

Finally, according to the least squares algorithm, the mobile receiver solves the coordinates by the corrected pseudo-range $\rho$. Let $\left(x_{u}^{k}, y_{u}^{k}, z_{u}^{k}\right)$ denote the corrected coordinates of the mobile station. Then, the pseudo-range of the mobile station is

$$
\rho=\sqrt{\left(x_{u}^{k}-x_{s}^{i}\right)^{2}+\left(y_{u}^{k}-y_{s}^{i}\right)^{2}+\left(z_{u}^{k}-z_{s}^{i}\right)^{2}}+c t_{u}^{k}
$$


where $t_{u}$ denotes the time-offset estimation.

\section{Edge Computing-based Differential Positioning Algorithm}

In the DCDP method, the data processing center computes the difference value. However, this process can cause delay and waste bandwidth. To resolve the disadvantages of the original DCDP method, this paper proposes the ECDP method, which exhibits low delay and provides mobility support. Moreover, the ECDP method does not affect the accuracy of the DCDP method.

\subsection{Edge Computing}

Edge computing refers to the enabling technologies that integrate the network, computing, storage, and application of the core capabilities and provide intelligent services at the edge of the network or near the edge of the data source [17]. Edge computing can meet key requirements of real-time business, data optimization, application intelligence, security and privacy protection [18].

Data are produced at the edge of the network rapidly; thus, data can be processed more efficiently at the edge of the network than at the central server [19]. In this paper, the reference station closest to the mobile receiver is used as the edge device to compute data instead of at the data center.

In the DCDP method, there are several principles to calculate the integrated result at the data center, including the minimum variance, weighted average and adjustment calculations.

The above methods reduce the risk of device damage due to the effect of multiple reference stations. However, these methods increase delay. Under this condition, the DCDP method cannot provide a rapid response. Therefore, this paper proposes using the nearest reference station at the edge of the network to compute the difference value. Under this scheme, low delay requirement is satisfied by finding the reference station with the fastest transmission.

\subsection{ECDP Method}

The user receiver receives data from the surrounding reference stations. With several base stations broadcasting their difference values, the mobile receiver begins to calculate its own coordinates until the received difference value is successfully obtained from the nearest base station. According to the distribution shown in Fig. 3, the ECDP method chooses the nearest base station BS1 to get the final difference value.

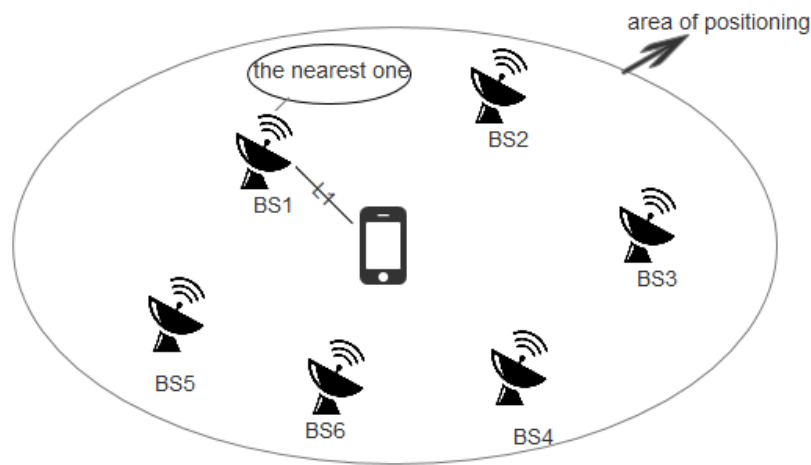

Fig. 3. ECDP method 
Assuming that the nearest base station has the highest transmission speed, and thus guarentee the stability when a mobile user switches from one to another nearest station. With several base stations broadcasting their difference values continuously, each time a mobile user sends a location request, the base station with the highest transmission speed is allowed. If the user moves to the range of another base station, the highest transmission will also change as the new nearest base station, thereby ensuring the most accurate positioning accuracy and the fastest transmission speed.

Due to the removal of the data center, this method decreases the transmission delay and calculation progress for the data center, and the new method also solves the problem of bandwidth waste. The new system is shown in Fig. 4.

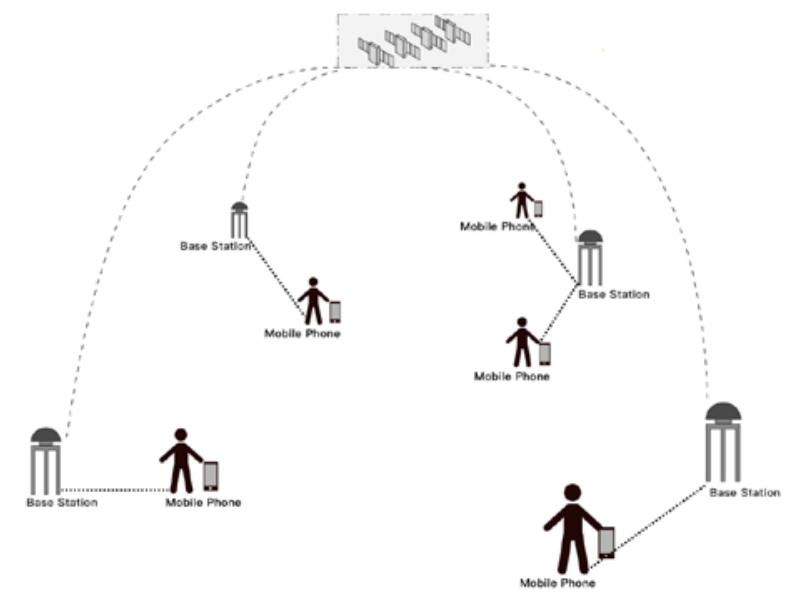

Fig. 4. ECDP system model

However, what cannot be ignored is that, despite of small probability, each base station has a lifetime or overload risk, and the progress of transmitting data is influenced by various uncertain parameters. All of these factors can cause the delay even the transmission failure. In the DCDP method, to reduce the above-mentioned risk, the DCDP method uses the data center to handle data, with the expense of increasing delay.

While for the ECDP method in a real positioning environment, a reasonable strategy is needed to prevent the nearest base station from being overloaded/damaged. For the case of damaged base station, the second nearest station can still transmit data to the user receiver at the system edge for calculation. But for the case of overloaded edge, when the nearest reference station is requested too much, exceeding the tolerance threshold, The ECDP method will trigger the shunting mechanism. When there is at least one available base station, the second nearest base station will transmit data to the user receiver at the edge of the network [9]. But when there is no available base station arround, since the reliability of the base station data is strongly related to the distance between stations, the data from outland base station cannot be directly forwarded. Therefore, the ECDP method performs edge computing using virtual base station within a short baseline range, where the virtual base station is generated by the nearest mobile receiver that can be connected to a real base station $[9,20]$. In the strict sense, the virtual reference station needs to obtain the error-related spatial error model, and then use the interpolation method to obtain the error-affected correction number based on the rough position of the mobile receiver. The strategy of the anti-overload edge is shown in Fig. $\mathbf{5}$. 


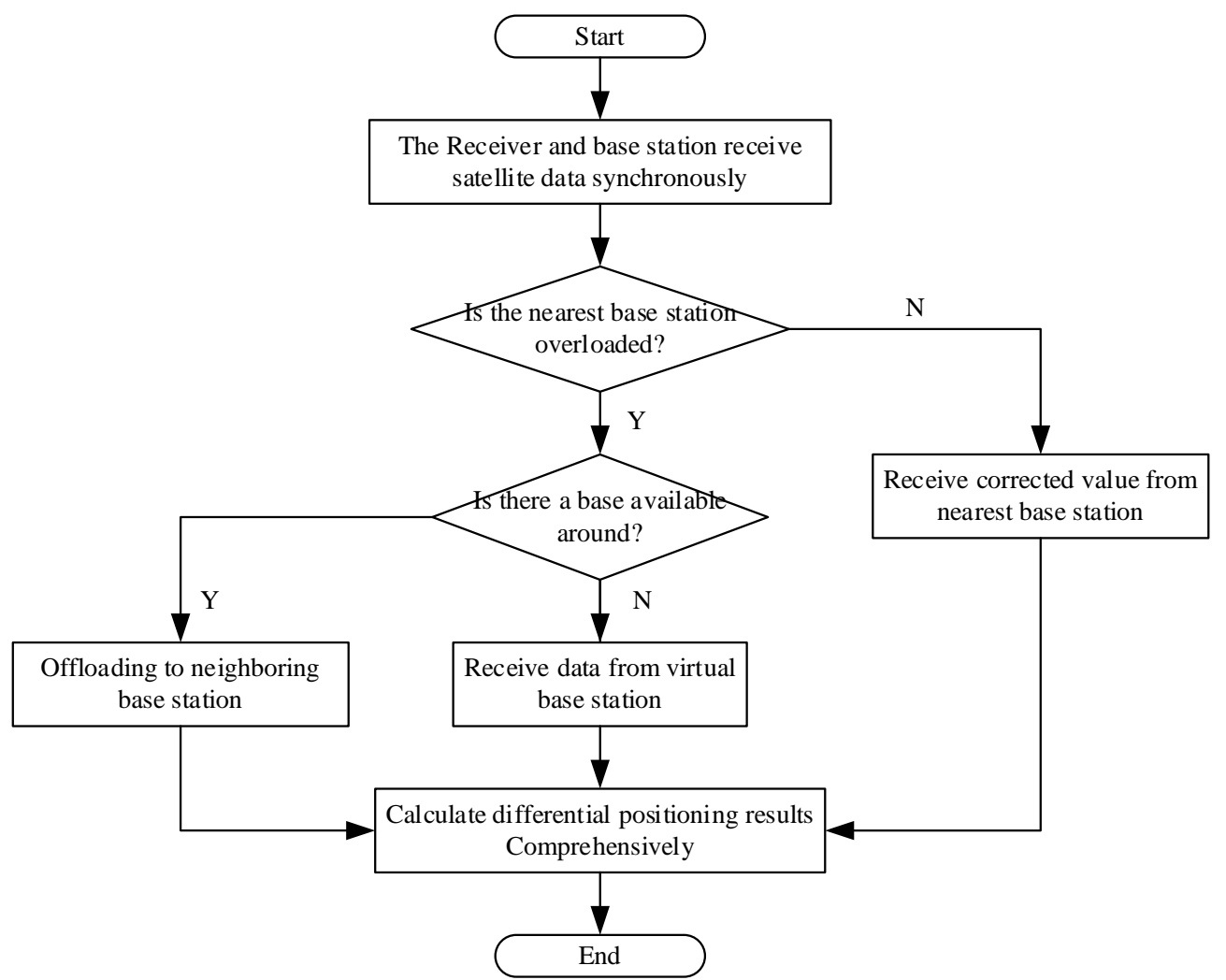

Fig. 5. Strategy of the anti-overload edge for ECDP method

\subsection{Pseudo-range-based Positioning Algorithm}

This paper uses the least squares iteration algorithm to determine the mobile receiver coordinates. The least squares iteration algorithm is widely used in the BDS because of its simplicity and maturity [21]. The basic principle used to obtain the optimal solution in the least squares iterative method is to minimize the sum of squares of the errors. When the difference value is obtained from the nearest base station, the solution algorithm of the mobile receivers uses the difference value to remove the common errors of the base station and mobile station. Most importantly, instead of transmitting the distance deviation to the data center and broadcasting to users, edge computing from the nearest reference station to the mobile receivers is used. The improved method decreases delay and avoids resource waste compared with the DCDP method. The details of the algorithm are as follows.

Let the approximate position of the user be $\left(\hat{x_{u}^{k}}, \hat{y_{u}^{k}}, \hat{z_{u}^{k}}\right)$; then, the true position of the user is $\left(x_{u}^{k}, y_{u}^{k}, z_{u}^{k}\right)$, and the pseudo-range can be denoted as follows:

$$
\begin{aligned}
\rho_{u}^{k} & =\sqrt{\left(x_{u}^{k}-x_{s}^{i}\right)^{2}+\left(y_{u}^{k}-y_{s}^{i}\right)^{2}+\left(z_{u}^{k}-z_{s}^{i}\right)^{2}}+c t_{u}^{k} \\
& =f\left(x_{u}^{k}, y_{u}^{k}, z_{u}^{k}, t_{u}^{k}\right)
\end{aligned}
$$

Similarly, the approximate pseudo-range is

$$
\begin{aligned}
\hat{\rho_{u}^{k}} & =\sqrt{\left(\hat{x_{u}^{k}}-x_{s}^{i}\right)^{2}+\left(\hat{y_{u}^{k}}-y_{s}^{i}\right)^{2}+\left(\hat{z_{u}^{k}}-z_{s}^{i}\right)^{2}}+c \hat{t_{u}^{k}} \\
& =f\left(\hat{x_{u}^{k}}, \hat{y_{u}^{k}}, \hat{z_{u}^{k}}, t_{u}^{k}\right)
\end{aligned}
$$


According to the Taylor expansion equation and with the non-linear term eliminated, the pseudo-range is expressed as (10) [22].

$$
\rho_{u}^{k}=\hat{\rho_{u}^{k}}-\frac{x_{s}^{i}-\hat{x_{u}^{k}}}{r^{\hat{i k}}} \Delta x_{u}^{k}-\frac{y_{s}^{i}-\hat{y_{u}^{k}}}{r^{i k}} \Delta y_{u}^{k}-\frac{z_{s}^{i}-\hat{z_{u}^{k}}}{r^{\hat{i k}}} \Delta z_{u}^{k}
$$

where $r^{\hat{i k}}=\sqrt{\left(x_{s}^{i}-x_{u}^{k}\right)^{2}+\left(y_{s}^{i}-y_{u}^{k}\right)^{2}+\left(z_{s}^{i}-z_{u}^{k}\right)^{2}}$

The position and time differences are given in (11).

$$
\begin{aligned}
& x_{u}^{k}=x_{u}^{k}+\Delta x_{u}^{k}, \quad y_{u}^{k}=y_{u}^{k}+\Delta y_{u}^{k}, \\
& z_{u}^{k}=z_{u}^{k}+\Delta z_{u}^{k}, \quad t_{u}^{k}=t_{u}^{k}+\Delta t_{u}^{k}
\end{aligned}
$$

For convenience, the following variables are introduced to simplify (11).

$$
\begin{aligned}
& \Delta \rho_{u}^{k}=\hat{\rho_{u}^{k}}-\rho_{u}^{k}, a_{x k}=\frac{x_{s}^{i}-\hat{x_{u}^{k}}}{\hat{\hat{k}}}, \\
& a_{y k}=\frac{y_{s}^{i}-\hat{y_{u}^{k}}}{\hat{r^{i k}}}, a_{z k}=\frac{z_{s}^{i}-z_{u}^{k}}{r^{i k}}
\end{aligned}
$$

Equation (10) can thus be written as follows [23].

$$
\Delta \rho_{u}^{k}=a_{x k} \Delta x_{u}^{k}+a_{y k} \Delta y_{u}^{k}+a_{z k} \Delta z_{u}^{k}+c t_{u}^{k}
$$

Let (13) be written in matrix form as (14) [23]

$$
\Delta \rho=H \Delta x
$$

where

$$
\Delta \rho=\left[\begin{array}{c}
\rho_{u}^{1} \\
\rho_{u}^{2} \\
\rho_{u}^{3} \\
\rho_{u}^{4}
\end{array}\right], H=\left[\begin{array}{llll}
a_{x 1} & a_{y 1} & a_{z 1} & 1 \\
a_{x 2} & a_{y 2} & a_{z 2} & 1 \\
a_{x 3} & a_{y 3} & a_{z 3} & 1 \\
a_{x 4} & a_{y 4} & a_{z 4} & 1
\end{array}\right], \Delta x=\left[\begin{array}{c}
\Delta x_{u}^{1} \\
\Delta y_{u}^{2} \\
\Delta z_{u}^{3} \\
\Delta t_{u}^{4}
\end{array}\right]
$$

Then, the solution of (14) is

$$
\Delta x=H^{-1} \Delta \rho
$$

Once the unknown variable is calculated, the true position $\left(x_{u}^{k}, y_{u}^{k}, z_{u}^{k}\right)$ can be calculated using (11).

The reference stations also use the least squares iteration algorithm to calculate the measured coordinates.

When obtaining the coordinates of the reference station, according to (4), the reference station calculates the deviation of the pseudo-range. Then, instead of transmitting the deviation to the data center, the reference station sends the data to the user receivers, which is called edge computing. The deviation of the nearest station is $\Delta \rho_{c e}^{j i}$, and its variation rate is $\Delta \dot{\rho}_{c e}^{j i}$. Therefore, the final pseudo-range is as shown in (17).

$$
\rho=\rho_{u}^{k i}+\Delta \rho_{c e}^{j i}+\Delta \dot{\rho}_{c e}^{j i}\left(t-t_{0}\right)
$$

Then, the corrected pseudo-range is used to correct the original result. 


\section{Numerical Results and Analysis}

This section presents the simulation and experimental results to demonstrate the efficiency and accuracy of the ECDP method. The ECDP method has several advantages compared with the DCDP method, such as low delay, low complexity and guaranteed accuracy.

\subsection{Data Pre-processing}

OEM6® Family Firmware which produced by NovAtel company, a subsidiary company affiliated with Sweden Hexagon Company, is used to collect the satellite data. The devices include the base station and mobile receiver. The software uses a high-precision receiver as the base station. And in order to meet the reliability of observation data, the paper considers the impact of the observation environment as much as possible in the experimental site selection. As shown in Fig.6, the site of the experiment is the playground of University of Science and Technology Beijing, which satisfies the following requirements for site selection.

- Be far away from water bodies, glass curtain walls and metal objects that are easy to produce multi-path effects.

- The elevation angle around the base station is not covered by more than 5 degrees: away from high voltage electricity Towers, radio transmitters, microwave stations and other electromagnetic interference areas.

- No high-voltage lines crossing from above.

- Be away from the railway along the line and other vibration-prone areas.

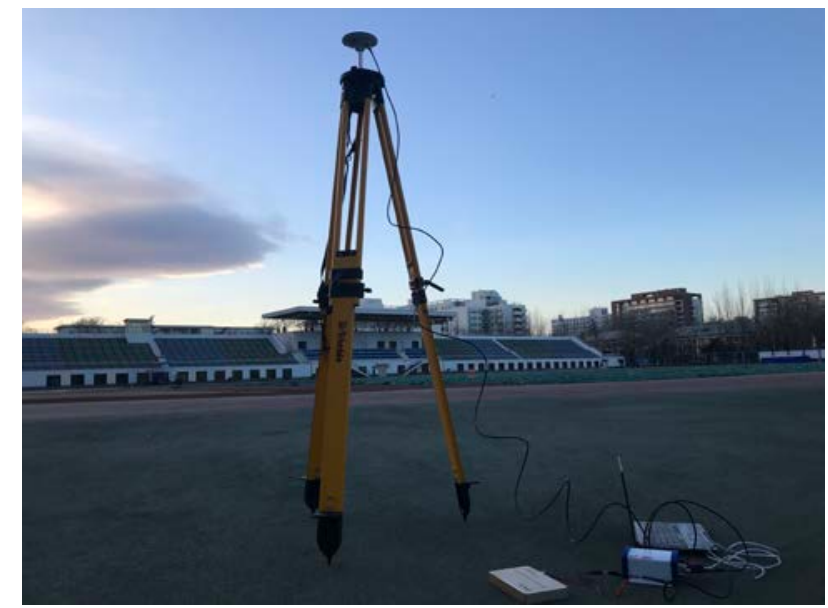

Fig. 6. Environment of BDS data collection

By connecting it to the antenna, the base station can obtain the precise coordinates of the receiver and BeiDou satellite. Then, the pseudo-range deviation $\Delta \rho_{c}^{j i}$ can also be obtained by sending data to the computer through a serial port. The mobile receiver can obtain the final coordinate results using the difference value from the nearest base station. The satellite status information can be obtained using the host computer software. As shown in Fig. 7, the current satellite conditions can be used to locate the coordinates of the receivers, meeting the positioning requirements. 


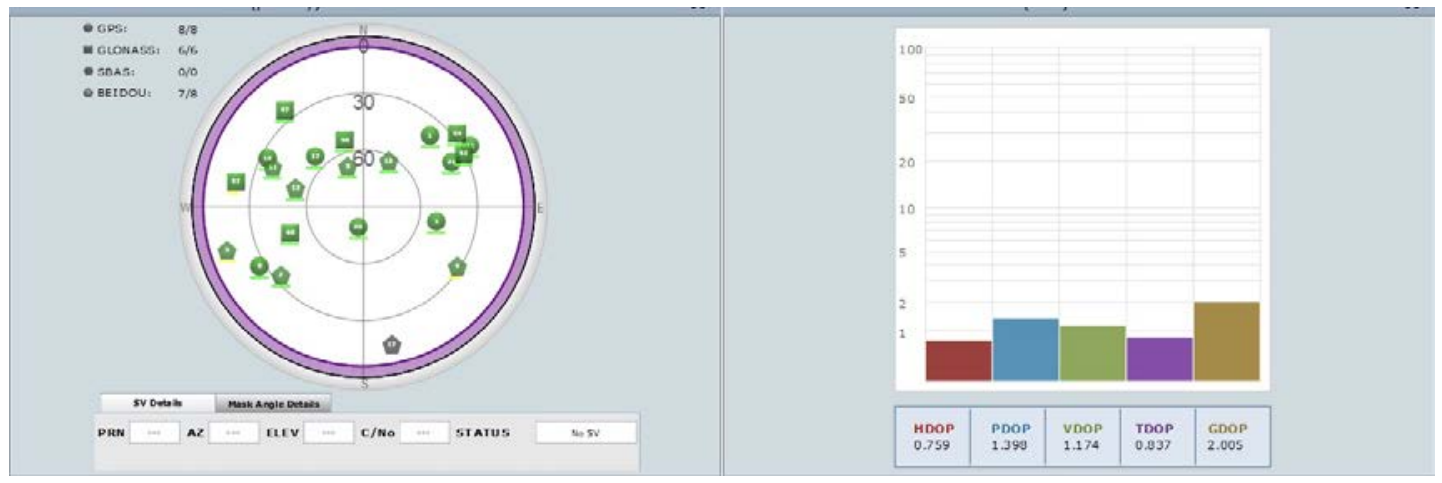

Fig. 7. Interface of the satellite states

By connecting the antenna, the OEM6® Family Firmware can receive the ephemeris parameters of the satellites. The satellite data can then be obtained with host computer software. The file of the base station can be obtained by sending command "Log satxyz2 ontime 1".

The base receiver can obtain the coordinates of visible satellites through the ephemeris parameters. All coordinates are then transformed into the CGCS2000 coordinate system of the BeiDou satellite system. For convenience, some satellite positioning data are listed systematically in Table 2.

Table 2. BeiDou satellite coordinates

\begin{tabular}{|c|c|c|c|}
\hline NO. & X & Y & Z \\
\hline BeiDou 1 & -32278726.6771 & 27095095.9105 & 1015818.6679 \\
\hline BeiDou 8 & 15634005.4651 & 37533334.7883 & 11574428.7805 \\
\hline BeiDou 4 & -39599343.7839 & 14454502.7898 & 707686.8018 \\
\hline BeiDou 7 & -22937935.1148 & 26515544.8530 & 23235942.8881 \\
\hline
\end{tabular}

\subsection{Positioning Accuracy Comparison}

As the quality of observed data is related to the geometric shape of satellites and receivers, the strength of precision is large. Thus, the position dilution of precision (PDOP) parameter is used to denote the relationship between the dimensional position accuracy and geometry navigation table. The PDOPs of the DCDP and ECDP methods are obtained and are shown in Fig. 8 and 9, respectively. The value of the PDOP is less than 2 during observation, regardless which method is used. 


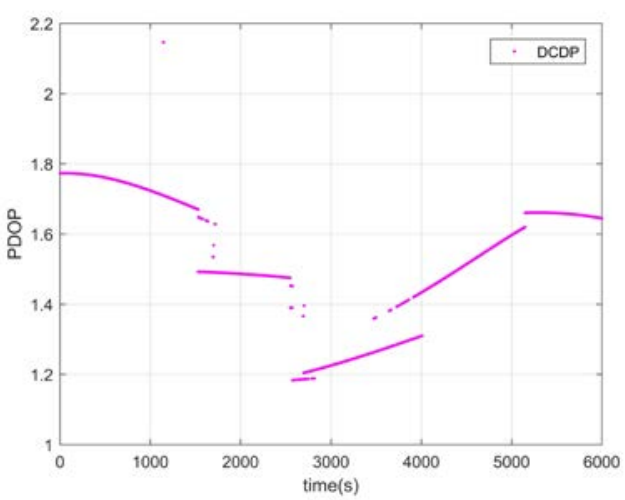

Fig. 8. PDOP of the DCDP method

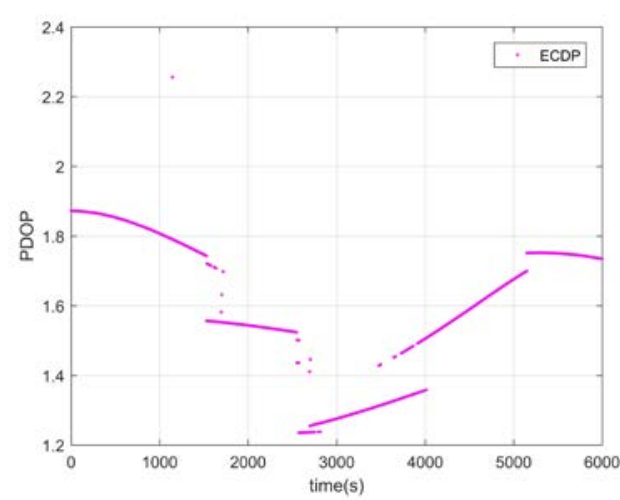

Fig. 9. PDOP of the ECDP method

In addition, the horizontal dilution of precision (HDOP) is an accuracy factor of the horizontal component, and the vertical dilution of precision (VDOP) is an accuracy factor of the vertical component. The relationship between the HDOP and VDOP is given in (18) [24].

$$
H D O P^{2}+V D O P^{2}=P D O P^{2}
$$

The HDOP and VDOP of the DCDP method are shown in Figure 8. The HDOP and VDOP of the ECDP method are shown in Fig. 11. These two figures illustrate that there is only a small difference between the two methods.
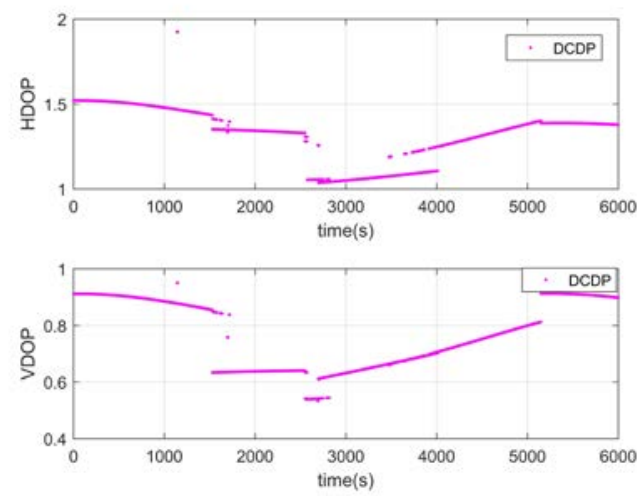

Fig. 9. HDOP and VDOP of the DCDP method
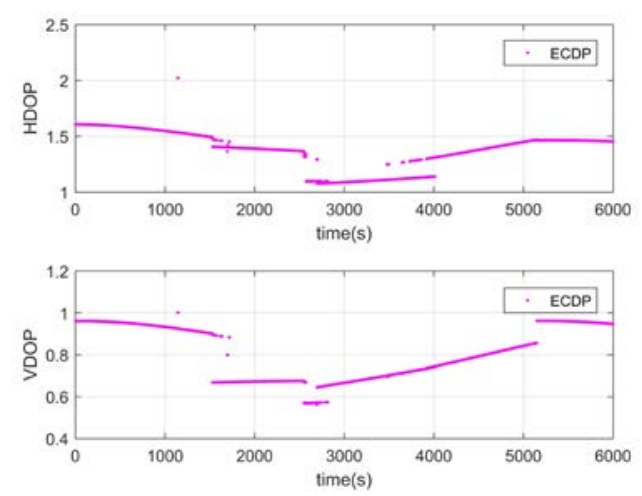

Fig. 10. HDOP and VDOP of the ECDP method

In the two different algorithms, the paper adopts a similar observation environment, which avoids other errors causing different interference to the results. From Fig. 8 to Fig. 11, the numerical performance is nearly uniform over time. 


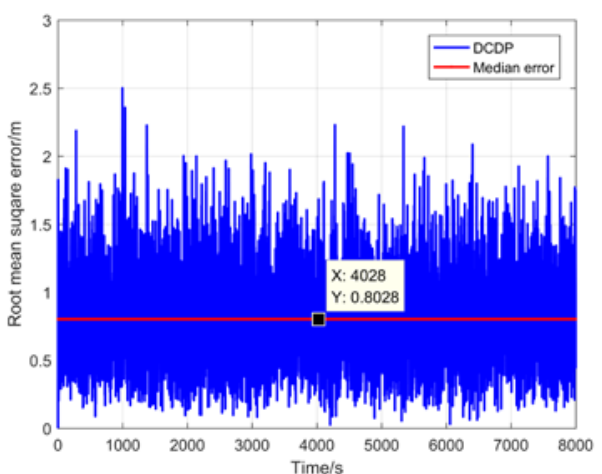

(a)Error of the DCDP method

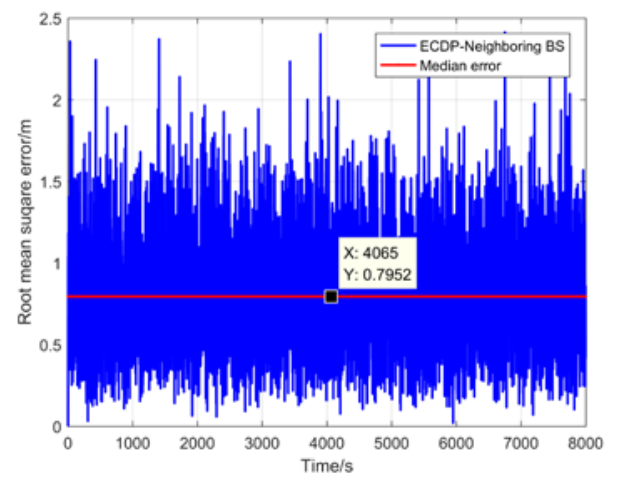

(c) Error of the ECDP method with neighboring BS

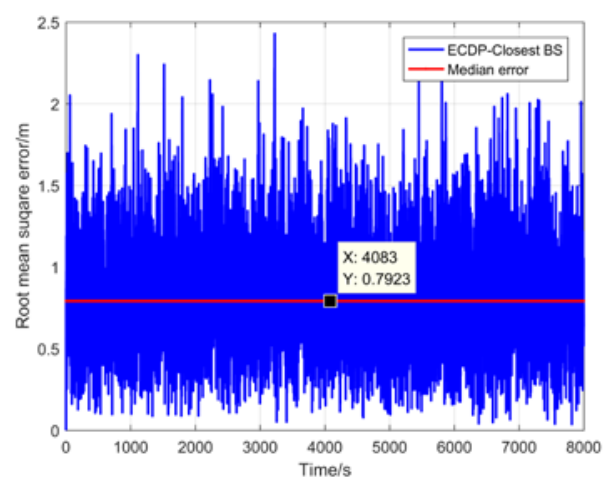

(b)Error of the ECDP method with closest BS

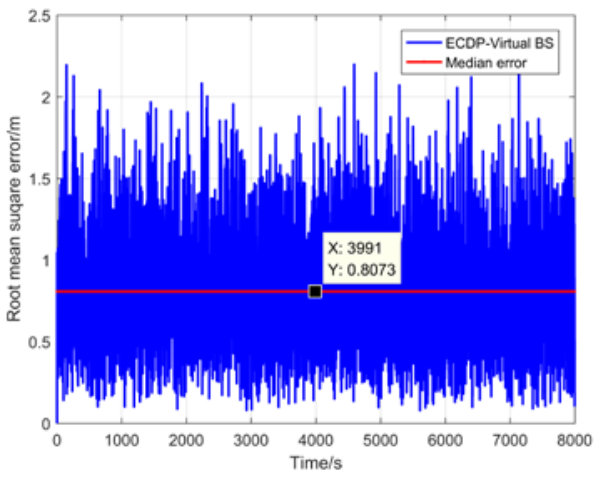

(d) Error of the ECDP method with virtual BS

Fig. 12. Errors of the DCDP and ECDP methods

Then, the receiver can obtain coordinates in the CGCS2000 coordinate system using the satellite coordinates combined with the pseudo-range difference algorithm. The positioning errors of the DCDP and ECDP methods are shown in Fig. 12. The accuracy of the two methods is similar, but the ECDP method should be separated to several cases. In order to compare the differences more quantitatively, the paper selects the median as an indicator to measure its error value. Through statistical calculations, it can be concluded that the median of DCDP method is $0.8028 \mathrm{~m}$ which is shown in Fig.12 (a). While for the ECDP method, it is shown with different cases in Fig.12 (b), (c) and (d). In the most case, when the nearest base station is not overloaded or damaged, the median of positioning accuracy is $0.7952 \mathrm{~m}$, improving $0.0105 \mathrm{~m}$ than DCDP method; When the neighboring base station was chosed, the median of positioning accuracy is $0.7923 \mathrm{~m}$, improving $0.0076 \mathrm{~m}$ than DCDP method; In rare cases, when the nearest base station is overloaded and there are no other available base stations around, the positioning accuracy of the virtual base station is $0.8073 \mathrm{~m}$, which is $0.0045 \mathrm{~m}$ more than DCDP method. All the cases demonstrate that the difference of ECDP and DCDP method is in the range of accidental errors, even in the case of uneven distribution of base stations, the virtual reference station can ensure reliable positioning accuracy, which provides guarantee for the expansion capability of the system. All these results demonstrate that the ECDP method can maintain the positioning accuracy and ensure positioning reliability. 


\subsection{Complexity Analysis}

There are $M$ satellites and $N$ reference stations participating in the differential positioning. Both methods are combined with the least squares iteration algorithm to perform $L$ iterations. The general least squares iteration algorithm must iterate for four variables, namely, three coordinates and the clock difference. Thus, the complexity is $O(4 M L) \approx O(M L)$. In addition, when a user requests positioning, there are $N$ reference stations participating in positioning in the DCDP method, whereas there is only one reference station in the ECDP method. Therefore, the complexity of the DCDP method is $O(M L)+O(N)$, whereas the complexity of the ECDP method is $O(M L)+O(1)$. Thus, the ECDP method is superior to the DCDP method in terms of complexity.

\subsection{Delay}

In the ECDP system, there is a single-hop communication delay between the user and base stations, whereas the DCDP system has a two-hop communication delay that includes transmitting data to the server and sending the final result to the mobile receiver. The known singular delay of transmitting data is $270 \mathrm{~ms}$. Thus, the delay of the ECDP method with transmitting data is approximately $270 \mathrm{~ms}$, and the delay of the DCDP method is approximately 540ms. Therefore, the ECDP method has a shorter response time during transmission; specifically, the delay is $270 \mathrm{~ms}$ shorter than that of the DCDP method.

Furthermore, in the DCDP method, after sending data to the server, the data center must handle the data of several base stations that consume time. The ECDP method does not need to consume this time.

Thus, the delay of the DCDP method is given in (19).

$$
t_{1}=2 t_{t}+t_{c}+t_{m}
$$

In (19), $t_{1}$ is the system delay; $t_{t}$ denotes the transmission time for one hop of data transmission; $t_{c}$ is the time that the data center handles correction values of the base stations; and $t_{m}$ is time that the mobile receiver solves the final positioning coordinates.

Similarly, the delay $t_{2}$ of the ECDP method is given in (20).

$$
t_{2}=t_{t}+t_{m}
$$

Therefore, the difference time between the DCDP and ECDP methods is as follows:

$$
\Delta t=t_{1}-t_{2}=t_{t}+t_{c}
$$

As noted above, the transmission delay of one hop $t_{t}$ is $270 \mathrm{~ms}$. Thus,

$$
\Delta t=270 m s+t_{c}
$$

Because $t_{c}$ increases with an increasing number of positioning users, the delay of DCDP method is not stable in the network. In the ECDP method, as the number of users increases, the delay is relatively stable and only depends on the transmission and solution times. The delay of the DCDP method is shown in Fig. 13, and the delay of the ECDP method is shown in Fig. 14. Fig. 14 illustrates that the delay of the ECDP method remains unchanged and less than 500ms with an increasing number of users. 


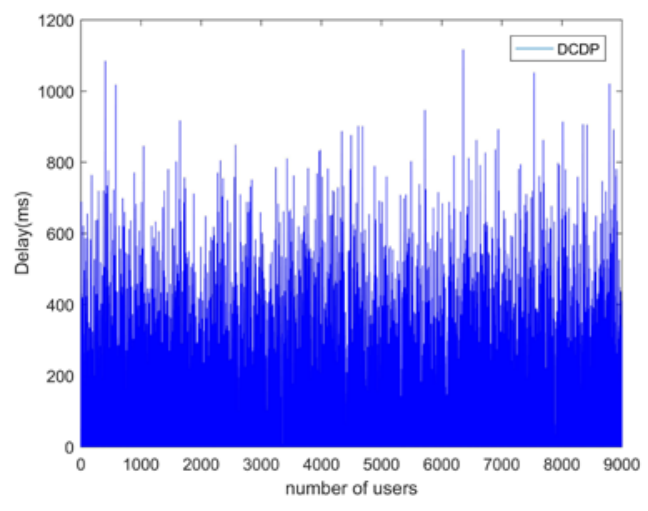

Fig. 13. Delay of the DCDP algorithm

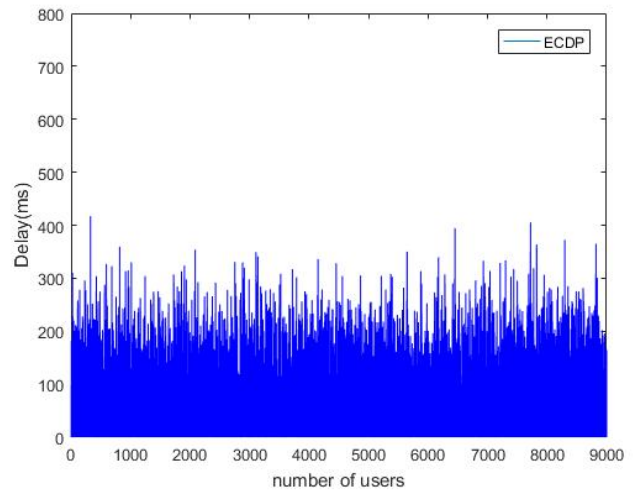

Fig. 14. Delay of the ECDP algorithm

Consequently, the ECDP method is better than the DCDP method in terms of delay. Moreover, the difference between the two methods will be greater with an increasing number of users. The ECDP method can efficiently solve a large number of positioning requests and responses.

\section{Conclusion}

This paper improves upon a general differential positioning method DCDP that uses a data center to process differential data. This process causes delay and bandwidth waste. Thus, this paper proposes an ECDP method to offload data center pressure with edge computing, which employs the closest reference station to the mobile receiver. In addition, simulation results demonstrate the ECDP method has low delay and guaranteed accuracy. The results provide insights for improving response times.

In future research, the positioning accuracy of the ECDP method will be improved. Accurate positioning results with a more rapid response will be achieved.

\section{References}

[1] Xianli Su, Xingqun Zhan, Mancang Niu, et al., "Receiver autonomous integrity monitoring availability and fault detection capability comparison between BeiDou and GPS," Journal of Shanghai Jiaotong University (Science), vol.19, no.3, pp. 313-324, June, 2014. Article (CrossRef Link).

[2] Shuanggen Jin, "Recent progresses on Beidou/COMPASS and other global navigation satellite systems (GNSS)-I," Advances in Space Research, vol. 51, no. 6, pp. 941, 2013. Article (CrossRef Link).

[3] OliverMontenbruck,Steigenberger Peterand HauschildAndré. "Broadcast versus precise ephemerides: a multi-GNSS perspective," GPS Solutions, vol. 19, no. 2, pp. 321-333, April, 2015. Article (CrossRef Link).

[4] Jianghui Geng,F. N. Teferle, et al., "Ambiguity resolution in precise point positioning with hourly data," GPS solutions, vol. 13, no. 4, pp. 263-270, September, 2009. Article (CrossRef Link).

[5] Dennis Odijk,Nandakumaran Nadarajah, Safoora Zaminpardaz and Peter J. G. Teunissen, "GPS, Galileo, QZSS and IRNSS differential ISBs: estimation and application," GPS solutions, vol. 21, no. 2, pp. 439-450, April, 2017. Article (CrossRef Link). 
[6] Erhu Wei, Hua Chai, Jingnan Liu and Zhiguo An, "On the generation algorithm of VRS virtual observations," Geo-spatial Information Science, vol. 10, no. 2, pp. 91-95, 2007. Article (CrossRef Link).

[7] Sihao Zhao, Xiaowei Cui, Feng Guan and Mingquan Lu, "A Kalman filter-based short baseline RTK algorithm for single-frequency combination of GPS and BDS," Sensors, vol. 14, no. 8, pp. 15415-15433, August, 2014. Article (CrossRef Link).

[8] Yun Chao Hu, Milan Patel, Dario Sabella, Nurit Sprecher and Valerie Young, "Mobile edge computing-A key technology towards 5G," ETSI White Paper, vol. 11, no. 11, pp. 1-16, September, 2015. Article (CrossRef Link).

[9] DimasSatria, Daihee Park, and Minho Jo, "Recovery for overloaded mobile edge computing," Future Generation Computer Systems, vol. 70, pp. 138-147, May, 2017. Article (CrossRef Link).

[10] Michael Armbrust, Armando Fox, et al., "A view of cloud computing," Communications of the ACM, vol. 53, no. 4, pp. 50-58, April, 2010. Article (CrossRef Link).

[11] M. B. Mollah,M. A. K. Azad, and A. Vasilakos, "Security and privacy challenges in mobile cloud computing: Survey and way ahead," Journal of Network and Computer Applications, 2017.

Article (CrossRef Link).

[12] WeipingJiang, RuijieXi, Hua Chen and Yugang Xiao, "Accuracy analysis of continuous deformation monitoring using BeiDou Navigation Satellite System at middle and high latitudes in China," Advances in Space Research, vol. 59, no. 3, pp. 843-857, February, 2017.

Article (CrossRef Link).

[13] Aykut, Nedim Onur, Engin Gülal, and Burak Akpinar, "Performance of Single Base RTK GNSS Method versus Network RTK," Earth Sciences Research Journal, vol. 19, no. 2, pp. 135-139, July, 2015. Article (CrossRef Link).

[14] Guorong $\mathrm{Hu}$, Victor Khoo, Pong Goh and Choi Law, "Performance of Singapore Integrated Multiple Reference Station Network (SIMRSN) for RTK positioning," GPS Solutions, vol. 6, no. 1-2, pp. 65-71, June, 2002. Article (CrossRef Link).

[15] Fuchs D L, Abraham C, van Diggelen F. Method and apparatus for forming a pseudo-range model: U.S. Patent 6,853,916[P]. 2005-2-8. Article (CrossRef Link).

[16] Lei, Wang, Chenglong Liu, Xuefeng Yang and Haijun Chen. "Three-Dimensional Adjustment Calculation for Free Station and Its Precision Evaluation in High-Speed Railway [J]," Journal of Geomatics Science and Technology, vol. 4, pp. 007, April, 2011. Article (CrossRef Link).

[17] Arif Ahmedand Ejaz Ahmed, "A survey on mobile edge computing," in Proc. of Intelligent Systems and Control (ISCO), 2016 10th International Conference on. IEEE, January 7-8, 2016. Article (CrossRef Link).

[18] Weisong Shi, and Schahram Dustdar, "The promise of edge computing," Computer, vol. 49, no. 5, pp. 78-81, May, 2016. Article (CrossRef Link).

[19] Weisong Shi, Jie Cao and Quan Zhang, "Edge computing: Vision and challenges," IEEE Internet of Things Journal, vol. 3, no. 5, pp. 637-646, June, 2016. Article (CrossRef Link).

[20] Tang W, Shen M, Deng C, et al., "Network-based triple-frequency carrier phase ambiguity resolution between reference stations using BDS data for long baselines[J],” GPS Solutions, 22(3): 73, 2018. Article (CrossRef Link).

[21] TianChi Yang, ChaoQun Yu, TianPeng Wang and Liang Jin, "A single observer location method under the scatter signals position disturbance situation: the constraint total least square method," Science China Information Sciences, vol. 54, no. 1, pp. 146-152, January, 2011. Article (CrossRef Link).

[22] Kang C. A., "Differential Dynamic Positioning Algorithm Based on GPS/Beidou[J]," Procedia engineering, 137: 590-598, 2016. Article (CrossRef Link).

[23] Goswami S. Global Positioning System[M]//Indoor Location Technologies. Springer, New York, NY, 2013: 51-63. Article (CrossRef Link).

[24] Yang Y X, Li J L, Xu J Y, et al., “Contribution of the compass satellite navigation system to global PNT users[J],” Chinese Science Bulletin, 56(26): 2813, 2011. Article (CrossRef Link). 


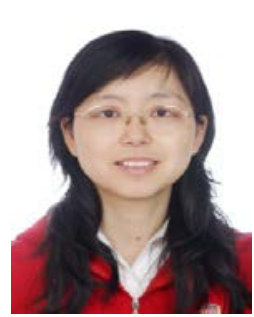

Lina Wang received M.S. and Ph.D. degrees in Communication and Information Systems from the Harbin Institute of Technology in 2001 and 2004, respectively. From 2010 to 2012, she was engaged in postdoctoral study at the University of Science and Technology Beijing. She is currently an associate professor in the Department of Communication Engineering, School of Computer and Communication Engineering, University of Science and Technology Beijing. Her research interests include space communications, BeiDou navigation systems, cognitive radio networks, fountain codes and game theory.

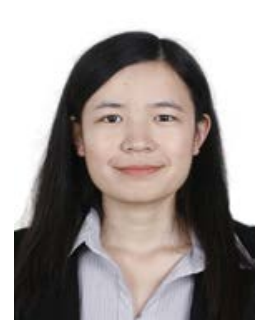

Linlin Li, received a B.S. degree at the University of Science and Technology Beijing in 2016. At present, she is pursuing her M.S. degree at the Department of Communication Engineering, School of Computer and Communication Engineering, University of Science and Technology Beijing. Her research interests are BeiDou positioning algorithms and edge computing.

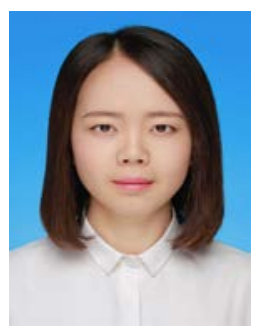

Rui Qiu, received a B.S. degree from the University of Science and Technology Beijing in 2017. At present, she is pursuing an M.S. degree at the Department of Communication Engineering, School of Computer and Communication Engineering, University of Science and Technology Beijing. Her research interests include BeiDou navigation algorithms. 